\title{
Paleoecología de Abies sp. en Sierra Bermeja (sur de la Península Ibérica) durante el Holoceno medio a partir del análisis pedoantracológico
}

\author{
Paleoecology of Abies sp. in Sierra Bermeja (southern Iberian Peninsula) \\ during the mid-Holocene from a pedoanthracological analysis
}

\author{
José Antonio Olmedo-Cobo ${ }^{\text {a*, }}$, Raquel Cunill-Artigas ${ }^{\text {b }}$, Emilio Martínez-Ibarra a , José Gómez-Zotano a

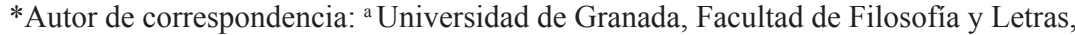 \\ Departamento de Análisis Geográfico Regional y Geografía Física, Campus Universitario Cartuja s/n, \\ Granada, España, tel.: 0034-626845825,jaolmedo@ugr.es, joseantonioolmedocobo@gmail.com \\ ${ }^{\mathrm{b}}$ Universidad Autónoma de Barcelona, Facultad de Filosofía y Letras, Departamento de Geografía, Barcelona, España.
}

\begin{abstract}
SUMMARY
This paper presents the first paleoecological results of the study of soil charcoals in the ultramafic massif of Sierra Bermeja (Betic Cordillera, southern Iberian Peninsula). Through the pedoanthracological analysis, a charcoal fossil register of Abies sp. has been obtained -with high certainty belonging to Abies pinsapo- in a small endorheic basin at the summit of Sierra Palmitera, a littoral mountainous appendix of Sierra Bermeja where the fir forests are extinct at present. Analyses results confirm the existence of a distribution area of Abies sp. more extensive in the past than currently in the geographical context of the Serrania de Ronda (western end of the Betic Cordillera). Dating of Abies sp. and other species found in the area of study, such as Pinus pinaster and Quercus sp., allowed theorizing about the local paleobotanical dynamic between 8,100 and 5,600 BP, largely solving the encysted dynamic and successional-ecologic controversy between broadleaf trees and coniferous trees as potential vegetation over ultramafic substrates under Mediterranean climate that occurred to that moment. This information should be considered as essential for the proper management of Abies pinsapo to achieve their preservation in the future given its cataloguing as a relict endangered species. The data also support the possible restoration of fir forests in those enclaves considered as potential ecological niches for these coniferous forests in Sierra Bermeja and also in the whole of the Betic Cordillera.
\end{abstract}

Key words: Abies pinsapo, soil charcoals, restricted distribution, paleoendemism, peridotites.

\section{RESUMEN}

En este trabajo se presentan los primeros resultados paleoecológicos del estudio de los carbones del suelo en el macizo ultramáfico de Sierra Bermeja (Cordillera Bética, sur de la Península Ibérica). Mediante el análisis pedoantracológico se ha obtenido un registro fósil de carbón de Abies sp. -con gran certeza perteneciente a Abies pinsapo- en una pequeña cuenca endorreica en la cumbre de Sierra Palmitera, un apéndice montañoso litoral de Sierra Bermeja en el que los abetales se encuentran actualmente extintos. Los resultados del análisis confirman la existencia, en el pasado, de un área de distribución de Abies sp. más extensa que la actual en el marco geográfico de la Serranía de Ronda (extremo occidental de la Cordillera Bética). Las dataciones de Abies sp. y de otras especies halladas en la localidad de estudio, como Pinus pinaster y Quercus sp., permiten teorizar sobre la dinámica paleobotánica local entre 8.100 y 5.600 AP, resolviendo en gran parte la enquistada controversia dinámica y sucesional-ecológica entre latifoliasconíferas como vegetación potencial sobre sustratos ultramáficos bajo clima mediterráneo que existía hasta ahora. Esta información debe ser considerada como esencial para la adecuada gestión de Abies pinsapo de cara a conseguir su preservación en el futuro dada su catalogación de especie relicta en peligro de extinción. Los datos obtenidos avalan, asimismo, la posible regeneración de los abetales en aquellos enclaves considerados nichos ecológicos potenciales para estos bosques en Sierra Bermeja y, en general, en el conjunto de la Cordillera Bética.

Palabras clave: Abies pinsapo, carbones del suelo, distribución restringida, paleoendemismo, peridotitas.

\section{INTRODUCCIÓN}

Abies pinsapo Boiss - popularmente conocido como "pinsapo"- es una de las especies arbóreas de mayor singularidad y valor ecológico de cuantas habitan en la
Península Ibérica, siendo el único abeto estrictamente mediterráneo presente en este territorio. Con un área de distribución disyunta, restringida al sector occidental de la Cordillera Bética, A. pinsapo crece en tres núcleos de montaña principales, cercanos pero separados entre sí, 
como son las sierras de las Nieves, Grazalema y Bermeja (provincias de Málaga y Cádiz). Estas áreas, por tanto, adquieren la categoría de refugio biogeográfico para esta especie, cuyos bosques se consideran formaciones de gran originalidad debido a su carácter paleoendémico y gran valor paisajístico y biogeográfico (Alba-Sánchez y López 2013, Gómez-Zotano et al. 2015). Se trata, además, de un árbol especialmente vulnerable al Cambio Climático, a los incendios forestales, al sobrepastoreo y a determinados agentes patógenos (principalmente hongos e insectos), que se han erigido en los principales factores de riesgo que amenazan su supervivencia. Por todo ello, A. pinsapo es un taxón incluido dentro del Catálogo Andaluz de Especies de Flora Silvestre amenazada del año 1994, habiéndose declarado como especie en peligro de extinción (EN) incluida dentro del Libro Rojo de la Flora Silvestre Amenazada de Andalucía (1999) y de la Lista Roja de la Flora Vascular de Andalucía (2005).

Dicho estatus amenazado se refleja en la preservación de los abetales béticos bajo distintas figuras de protección, como son los Parques Naturales y Reservas de la Biosfera de la UNESCO e Intercontinental del Mediterráneo de Sierra de las Nieves y Sierra de Grazalema, y el Paraje Natural de los Reales de Sierra Bermeja. Bajo el amparo de estos espacios protegidos se trabaja activamente para la conservación de sus hábitats y de la fauna y flora silvestres presentes en estos, siendo la lucha contra los principales factores que amenazan la supervivencia de la especie una cuestión primordial. A escala europea, además, el reconocimiento de su fragilidad y necesidad de protección llevó a la declaración de estos bosques como el Hábitat de Interés Comunitario "9520 Abetales de Abies pinsapo Boiss." a partir de la Directiva 92/43/CEE de la Comisión Europea para el Medio Ambiente, quedando consecuentemente estas florestas integradas en la propuesta de Lugares de Importancia Comunitaria -LIC-y en el marco de la Red Ecológica Europea NATURA 2000.

La importancia ecológica y estatus amenazado de $A$. pinsapo ha favorecido que en las últimas décadas sea una de las especies más estudiadas de la flora mediterránea. De este modo, dicho abeto ha centrado la atención de gran número de científicos y de las distintas administraciones encargadas de la gestión ambiental en el sur de España con el objeto de procurar su conservación. Como resultado lógico de este elevado interés, el conocimiento sobre la especie en la actualidad es notable, como también lo es de su historia reciente (siglo XVI en adelante). Sin embargo, existe una carencia casi absoluta de fuentes de información paleoecológicas, más allá de los registros fósiles de polen de Abies sp. encontrados en distintos emplazamientos sur-ibéricos. En este sentido, de acuerdo con Alba-Sánchez y López (2013), aunque el conocimiento paleobiogeográfico de $A$. pinsapo está experimentando importantes avances en los últimos años, faltan análisis paleoecológicos que complementen los escasos estudios polínicos que se han realizado hasta la fecha en las serranías béticas refugio del pinsapo.
A partir de la escasa información paleoecológica disponible, en esta investigación se plantea como hipótesis de partida la existencia de un área de distribución pretérita de A. pinsapo más amplia que la actual en el sur de la Península Ibérica dada la existencia de biotopos potenciales para esta especie que hoy día no están ocupados por ella, corolario que también se justifica con los mapas de potencialidad de A. pinsapo desarrollados por diversos autores, siendo el trabajo de Alba-Sánchez y López (2013) el más reciente y detallado. Para corroborar o no dicho presupuesto inicial, y como fórmula para subsanar la deficiencia de conocimiento antedicha, el presente trabajo aborda un análisis pedoantracológico - estudio de los carbones del suelo- en una localidad de gran particularidad coincidente con una pequeña cuenca endorreica localizada en el macizo ultramáfico de Sierra Bermeja, uno de los tres núcleos en los actualmente perviven abetales mediterráneos en el extremo occidental de la Cordillera Bética. Los resultados obtenidos muestran una gran riqueza de carbón en el registro fósil de la cuenca endorreica, destacando entre las especies identificadas el hallazgo de carbón de Abies sp. con una antigüedad superior a 5.000 años.

Los datos colectados ofrecen una valiosa información paleoecológica de las dinámicas naturales en la localidad de estudio durante el Holoceno medio y permiten discutir la hipótesis de partida. Esta información puede permitir el desarrollo e implementación de una nueva estrategia para la preservación y regeneración de $A$. pinsapo apoyada en el conocimiento de su paleo-evolución. Unas nuevas posibilidades de gestión proteccionista que han de permitir afrontar con mayores garantías de éxito el gran reto de la supervivencia de este abeto mediterráneo en el futuro ante el escenario de Cambio Global que lo amenaza.

\section{MÉTODOS}

Área de estudio. Sierra Bermeja es un macizo montañoso de mediana altitud (Encinetas $1.473 \mathrm{~m} \mathrm{snm}$, Los Reales $1.449 \mathrm{~m} \mathrm{snm}$ ) situado en el extremo occidental de la Cordillera Bética (sur de la Península Ibérica), que constituye el mayor afloramiento peridotítico del conjunto petrográfico de la Serranía de Ronda, y uno de los más grandes del mundo con $300 \mathrm{~km}^{2}$. Por tanto, se trata de un ámbito montañoso que destaca por su litología ya que la peridotita es una roca ígnea ultramáfica de gran dureza y densidad, compuesta por minerales ferromagnésicos $(>40 \%$ olivino) que, una vez alterados, reciben el nombre genérico de serpentinas. Sobre este material se desarrollan suelos con una elevada composición de metales pesados sin función biológica conocida $(\mathrm{Cr}, \mathrm{Ni}, \mathrm{Co}, \mathrm{Cu})$, que incluyen además limitaciones excepcionales de nutrientes esenciales tales como nitrógeno, fósforo y/o potasio, así como de cationes básicos, además de una baja proporción de $\mathrm{Ca}^{2+} / \mathrm{Mg}^{2+}$, lo que, en su conjunto, conlleva disfunciones fisiológicas en los organismos sésiles (plantas) y un carácter xerófilo de los suelos, con alta susceptibilidad a la erosión (Mota 
et al. 2008). Asimismo, la posición estratégica entre dos continentes ha convertido a este macizo en un refugio para algunos taxones de la flora del Terciario que habían emigrado de Europa a África y viceversa (Alba-Sánchez y López 2013).

En el seno de Sierra Bermeja, y como uno de sus principales apéndices montañosos, se localiza Sierra Palmitera (1.473 m snm) (figura 1), en donde se ubica la localidad objeto de estudio, correspondiente a una pequeña cuenca endorreica de, aproximadamente, 30 hectáreas de superficie, situada a $1.364 \mathrm{~m} \mathrm{snm}\left(36^{\circ} 35^{\prime} 53^{\prime \prime} \mathrm{N}, 5^{\circ} 03^{\prime} 21^{\prime \prime} \mathrm{W}\right)$ (figura 2), formada sobre una base litológica peridotítica y sustentadora de suelos serpentínicos, y en donde $A$. pinsapo está ausente hoy día. De acuerdo con Gómez-Zotano et al. (2015), dada la naturaleza ultramáfica de esta montaña litoral, la cuenca se considera una excepcionalidad geomorfológica para el conjunto de los afloramientos peridotíticos conocidos, a la vez que presenta una importante acumulación de sedimentos (arcillas) en el fondo, razón por la cual se la ha elegido como área de estudio ya que posibilita el análisis paleo-ecológico de la vegetación a partir de los carbones depositados en el suelo serpentinítico, de tipo regosol. Su altitud y cercanía al Estrecho de Gibraltar favorecen un clima mediterráneo de media montaña notablemente húmedo (PMA en torno a 1.000-1.300 mm, ombroclima húmedo), con destacable criptoprecipitación y temperaturas medias anuales de $13-15{ }^{\circ} \mathrm{C}$ (termotipo mesomediterráneo).

Desde un punto de vista biogeográfico, el área de estudio pertenece según Rivas-Martínez (2011) al distrito Bermejense del sector Rondeño de la provincia corológica Bética (Región Mediterránea, Reino Holártico), cuya vegetación se caracteriza por la existencia de dos grandes dominios forestales: los pinares edafoxerófilos de pino resinero (Pinus pinaster Aiton), extendidos por buena parte de Sierra Bermeja, y los abetales climatófilos de A. pinsapo, limitados actualmente a las cimas de Los Reales, Abanto, Plaza de Armas y Corona. Estos bosques identifican, respectivamente, las series de vegetación exclusivamente bermejenses Querco cocciferae-Pineto pinastri S. (encabezada por la asociación de pinar-coscojar Querco-Pinetum pinastri Cabezudo, Nieto-Caldera y Pérez-Latorre

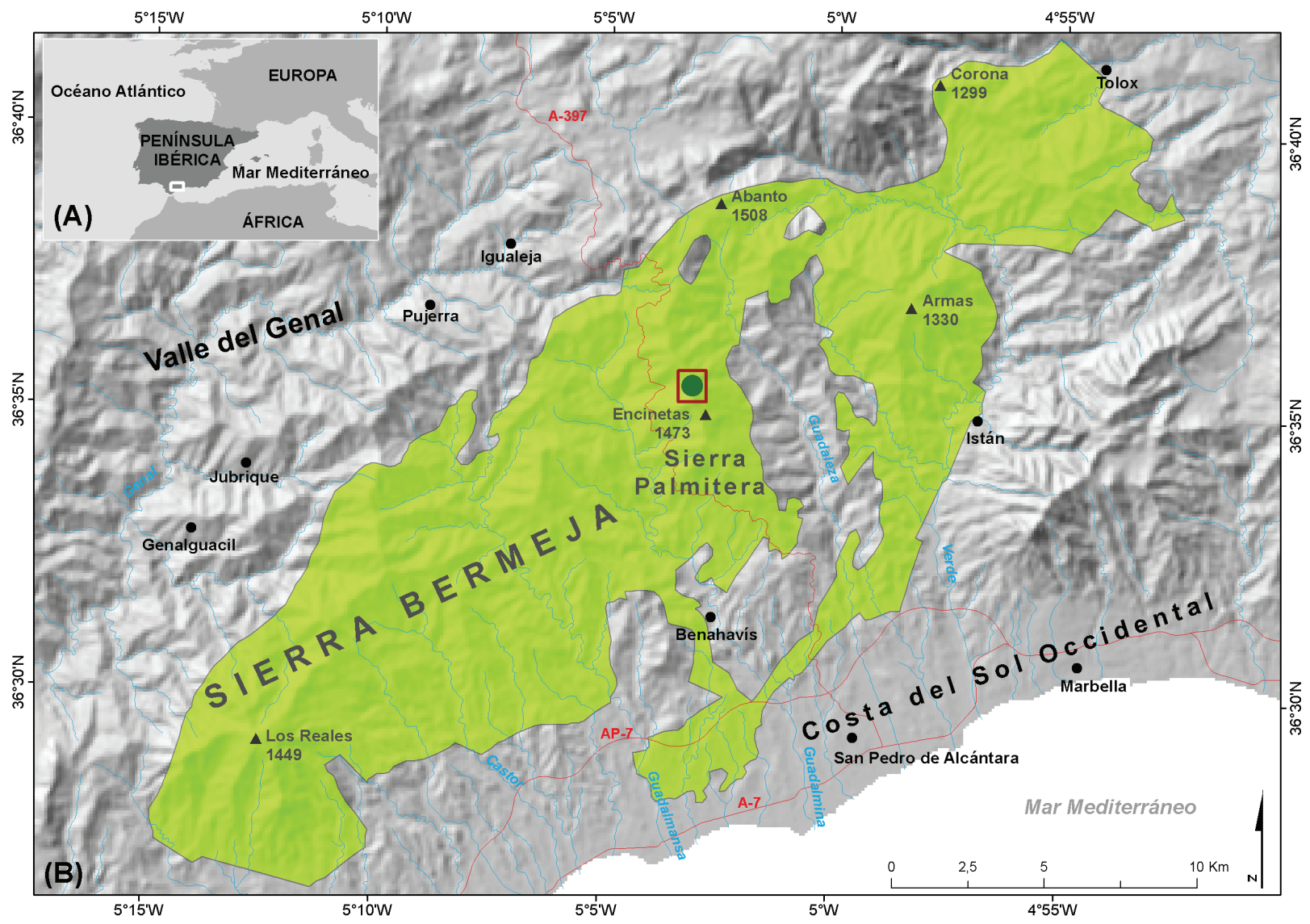

Figura 1. Localización del área de estudio en el sur de la Península Ibérica (A) y en Sierra Bermeja (B). En color verde se marca el dominio ultramáfico de las peridotitas.

Location of the study area in the south of the Iberian Peninsula (A) and in Sierra Bermeja (B). Green color marks the ultramafic domain of the peridotites. 


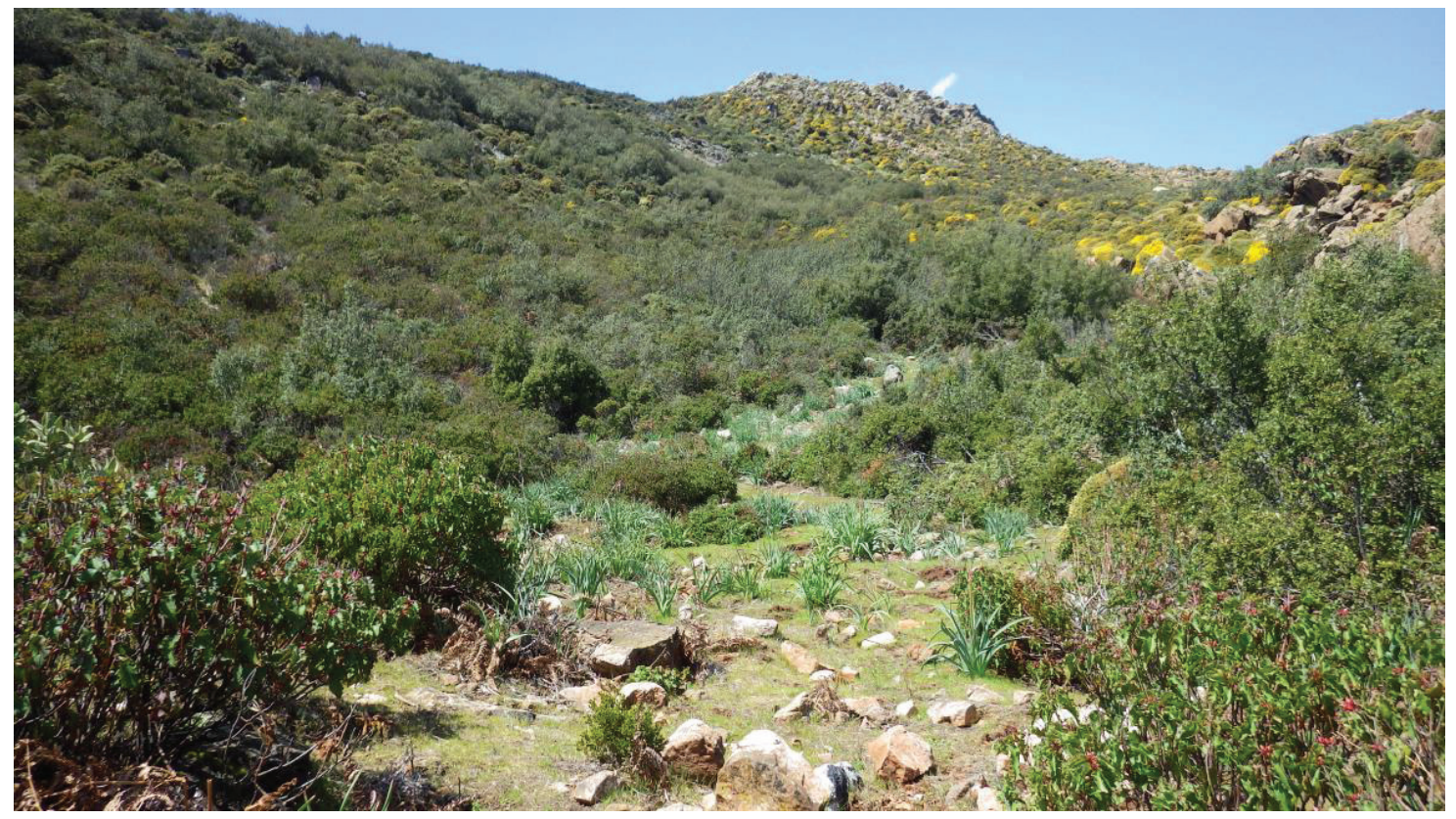

Figura 2. Aspecto parcial de la cuenca endorreica de Sierra Palmitera objeto de análisis. En primer plano se observa el fondo de la depresión donde se llevó a cabo el sondeo pedoantracológico.

Partial aspect of the endorheic basin of Sierra Palmitera which is object of analysis. The bottom of the depression, where the pedoanthracological sounding is conducted, is observed in the foreground.

1989) y Bunio macucae-Abieteto pinsapo $S$. [encabezada por la asociación de abetal Bunio macucae-Abietetum pinsaponis (Asensi et Rivas-Martínez 1976) Rivas-Martínez 1987]. En la localidad de estudio aparecen dos comunidades de matorral que forman parte, habitualmente, de la sucesión vegetal teórica que acompaña a estos abetales serpentinícolas bermejenses, como son los piornales de aulaga vaquera (Ulex baeticus Boiss.) y tojos alfileteros [Genista hirsuta Vahl ssp. lanuginosa (Spach) Nyman], y los jarales de jara macho (Cistus populifolius L.), que en su conjunto integran la asociación Genisto lanuginosaeCistetum populifolii Asensi et Díez-Garretas 1992, exclusiva de Sierra Bermeja y representativa de la degradación de las etapas de prebosque de orla del abetal. Asimismo, destaca la aparición de un bosquete de encinas (Quercus rotundifolia Lam.) relicto en el contexto de Sierra Bermeja, de carácter cerrado y talla media, con ejemplares de porte arbustivo-arborescente; se trata de una tesela con vegetación que, a falta de un estudio florístico detallado, no es posible asimilar de momento a ninguno de los encinares béticos definidos desde el punto de vista fitosociológico. Dentro de este contexto vegetal, la flora, tal y como recoge en síntesis Gómez-Zotano et al. (2014), se ve drásticamente condicionada a causa del especial ambiente edáfico (conocido como síndrome de serpentina), lo que favorece la presencia de numerosos taxones exclusivos de los suelos serpentínicos y, en contrapartida, se caracteriza por la exclusión de la mayoría de las especies de las formaciones mediterráneas circundantes.
Metodología. El método para llevar a buen término la investigación se ha basado en la pedoantracología, disciplina científica que, como el resto de sus afines paleoecológicas, ofrece una valiosa información de la geohistoria ambiental y de la paleobiogeografía de un territorio. A partir del análisis del registro fósil vegetal (granos de polen, esporas, semillas, frutos, micro y macrocarbones, microfósiles polínicos, fitolitos, etc.) se puede conocer con gran nivel de detalle los distintos ciclos y dinámicas temporales y espaciales de tipo climático y forestal que han afectado a una región determinada. La importancia de este tipo de estudios radica en dos puntos esenciales (Alcalde et al. 2006): en primer lugar, la posibilidad de modelizar los ecosistemas forestales del pasado y, en segundo, la capacidad para estudiar la dinámica temporal de estos en distintas fases paleohistóricas, precisando la naturaleza de las principales perturbaciones (climáticas y/o antrópicas) acaecidas.

La pedoantracología, en concreto, se basa en el estudio de los carbones vegetales del suelo, lo que permite la reconstrucción de la paleo-dinámica de la vegetación arbórea y leñosa incendiada con gran precisión espacial (Carcaillet y Talon 1996, Talon et al. 1998) ya que, a diferencia de los análisis palinológicos (polen), los estudios antracológicos aseguran la presencia in situ de la especie fuente del carbón.

En este contexto metodológico general, en la presente investigación se han llevado a cabo dos fases principales de trabajo, una en campo y otra en laboratorio. En primer lugar, el trabajo de campo ha consistido en la toma 
de muestras de suelo en el fondo de la cuenca objeto de estudio (localidad "PALMI"), ya que se consideró que este era el punto más idóneo para encontrar un registro fósil de carbón más amplio. Para ello, se realizó un sondeo abriendo una fosa de una profundidad de $80 \mathrm{~cm}$ hasta alcanzar las primeras evidencias físicas de roca madre. En dicho corte edáfico se identificaron cinco niveles de muestreo (I, II, III, IV, V), delimitados a partir de la descripción de los horizontes del suelo, tomándose en cada uno de ellos una muestra de suelo de aproximadamente $5 \mathrm{~kg}$ de peso (cinco muestras de suelo en conjunto).

El trabajo de laboratorio, en segundo lugar, ha consistido en la separación e identificación del carbón a partir del procedimiento descrito por Carcaillet y Thinon (1996) y Talon et al. (1998). Para ello, se procedió al tamizado en agua de las muestras de suelo recogidas en campo. El tamaño de las mallas de tamizado ha sido de 5, 2 y $0,8 \mathrm{~mm}$ $y$, por tanto, los tamaños de los carbones resultantes han sido de $>5 \mathrm{~mm}, 5$ a $2 \mathrm{~mm}$ y 2 a $0,8 \mathrm{~mm}$. Posteriormente, se llevó a cabo la selección manual del carbón y a su separación de la fracción mineral sobrante en cada tamiz con la ayuda de una lupa binocular. A continuación, se realizó la identificación taxonómica de un máximo de 100 carbones por cada tamaño de malla $(5,2$ y $0,8 \mathrm{~mm})$ de cada nivel de muestreo (I, II, III, IV, V). Para esta discriminación de especies, se utilizó un microscopio episcópico (aumentos de 100x, 200x y 500x), tomando como referencia de base diferentes atlas de anatomía de maderas y carbones, así como la propia antracoteca del Laboratorio de Geografía Física de la Universidad de Granada (España).

Por último, las muestras seleccionadas de las distintas especies identificadas (10 para la localidad de estudio) se enviaron para su datación mediante AMS (prueba del ${ }^{14} \mathrm{C}$ ) al laboratorio especializado Poznan Radiocarbon Laboratory (Poznan, Polonia). Dichas muestras tuvieron que ser pesadas, previamente a su envío, en una báscula de precisión para comprobar que, en todos los casos, presentasen el peso mínimo necesario - $3 \mathrm{mg}$ - para su datación mediante la técnica de radio-carbón ${ }^{14} \mathrm{C}$ (téngase en cuenta que la mayor parte de las muestras analizadas no alcanzaba un peso de $3 \mathrm{mg}$ ). Las dataciones obtenidas se han calibrado con el programa Oxcal 4.2 y la base de datos IntCal13 (Reimer et al. 2013), 2 sigma (95\% probabilidad).

\section{RESULTADOS}

Análisis de la antracomasa encontrada y especies identificadas. Tal y como se representa en la figura 3A, en todos los niveles de muestreo (I, II, III, IV y V) se ha encontrado carbón en cantidad importante. En I, III y V (figura 3B) las tasas de antracomasa (mg de carbón por kg de muestra se suelo) oscilan entre 4.000 y algo más de $4.600 \mathrm{mg} \mathrm{kg}^{-1}$, que se pueden considerar valores relativamente elevados. El nivel IV presenta una tasa de antracomasa superior, ligeramente por encima de $10.800 \mathrm{mg} \mathrm{kg}^{-1}$. Sin embargo, es el nivel II -coincidente con una profundidad en el suelo de 10 a 18-20 cm- en donde la tasa de antracomasa es mayor, con un valor que supera los $112.000 \mathrm{mg} \mathrm{kg}^{-1}$ que, en este caso, prácticamente no encuentra parangón en análisis pedoantracológico alguno.

Respecto a las especies identificadas, han sido dos los taxones principales, correspondientes a Quercus sp. y, en menor medida, a $P$. pinaster (figura $3 \mathrm{C}$ y cuadro 1 ). A Quercus sp. corresponde la mayor parte de los carbones encontrados en I, II, III y V (70-95\%); por su parte, $P$. pinaster se encuentra modestamente representado en I, III y V (5-15 \%), siendo la especie mayoritaria en IV (60 $\%$ ), único nivel en el que el predominio de carbones encontrados no corresponde a Quercus sp. En su conjunto, Quercus sp. y P. pinaster representan entre el $78 \%$ y el

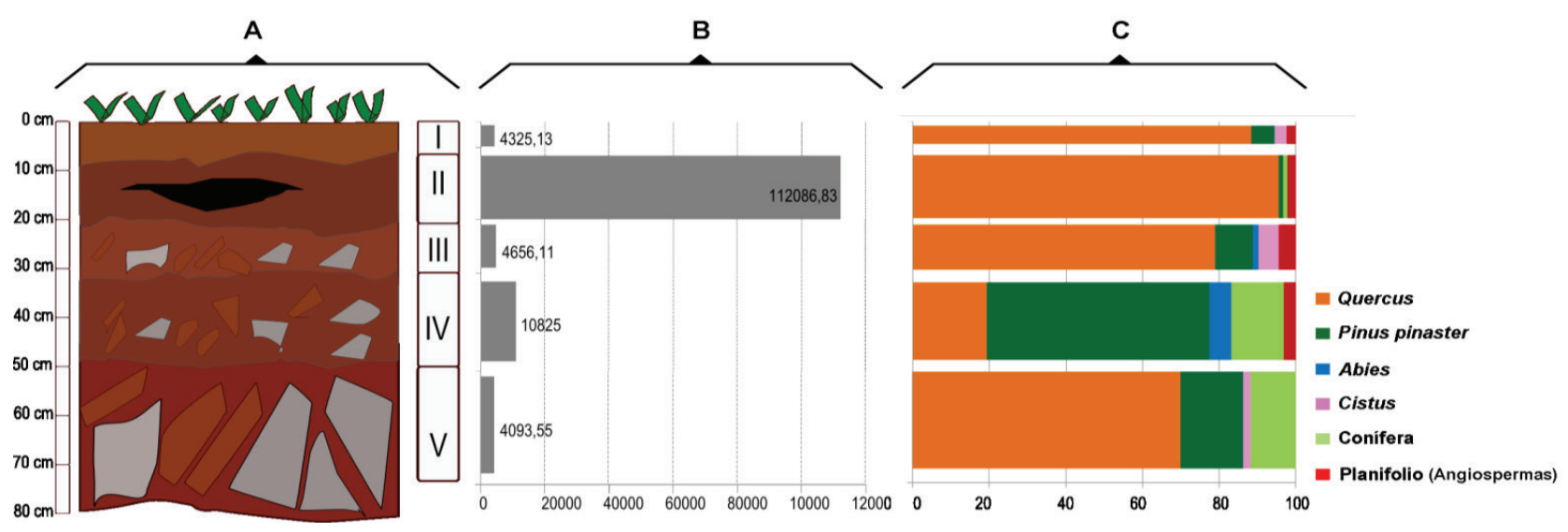

Figura 3. Resultados por nivel de muestreo: A) perfil del suelo, B) antracomasa específica por nivel (mg $\left.\mathrm{kg}^{-1}\right)$ y C) taxones identificados en porcentaje (proporción calculada excluyendo los carbones vitrificados y no identificados).

Results by sampling level; A) Soil profile; B) Specific anthracomass by level ( $\left.\mathrm{mg} \mathrm{kg}^{-1}\right)$; C) Identified taxa in \% (proportion calculated excluding the vitrified and unidentified charcoals). 
Cuadro 1. Resultados de la identificación taxonómica: número de fragmentos de carbón analizados por nivel de muestreo.

Results of the taxonomic identification: number of charcoal fragments analyzed by sampling level.

\begin{tabular}{lrrrrrr}
\hline \multirow{2}{*}{ Especies } & \multicolumn{6}{c}{ Nivel de muestreo } \\
\cline { 2 - 7 } & I & II & III & IV & V & Total \\
\hline Quercus sp. & 143 & 167 & 105 & 24 & 77 & 516 \\
Pinus pinaster & 10 & 2 & 13 & 72 & 18 & 115 \\
Abies sp. & 0 & 0 & 2 & 7 & 0 & 9 \\
Cistus sp. & 5 & 0 & 7 & 0 & 2 & 14 \\
Angiosperma & 4 & 4 & 6 & 4 & 0 & 18 \\
Gimnosperma & 0 & 2 & 0 & 17 & 13 & 32 \\
No identificado & 11 & 5 & 17 & 6 & 42 & 81 \\
Vitrificado & 77 & 74 & 93 & 47 & 66 & 357 \\
\hline Total & 250 & 254 & 243 & 177 & 218 & 1.142 \\
\hline
\end{tabular}

$96 \%$ del total de los fragmentos de carbón analizados en los distintos niveles de muestreo (un total de 631 de 1.142 muestras, lo que representa el 55,2\%).

Otros taxones presentes pero escasamente representados en el registro fósil de carbón han sido Abies sp. y Cistus sp., que, además, no aparecen en todos los niveles de muestreo (el primero únicamente está presente en el nivel IV y el segundo aparece puntualmente en I, III y V). También ha resultado escaso el número de muestras identificadas a nivel de angiosperma-gimnosperma (un total de 50 muestras en el conjunto de todos los niveles, es decir, un 4,4\% del total), así como el de carbones no identificados (39 muestras en I, II, III y IV, 3,4 \% del total) a excepción del nivel V, donde las muestras no identificadas han alcanzado las 42 (19,2 \% respecto al total de dicho nivel). En ambos casos, angiospermas-gimnospermas y carbones no identificados, se ha tratado de muestras en las que solo ha sido posible reconocer alguna de las características anatómicas del carbón, sin que ello haya permitido llegar a una identificación de mayor detalle o a ninguna aproximación taxonómica válida. Mayor significación han tenido las muestras de carbón cuya identificación ha sido imposible por hallarse completamente vitrificadas, es decir, sin posibilidad alguna de reconocer características definitorias de una u otra especie, género o división vegetal. En concreto, se ha encontrado un total de 357 muestras vitrificadas, lo que representa un 31,2 $\%$ del total, habiendo en este caso una relativa homogeneidad en la cantidad de carbones vitrificados respecto al total de muestras analizadas en cada nivel de muestreo (mínimo de $26,5 \%$ en IV y máximo de $38,2 \%$ en III).

Dataciones. De las 654 muestras identificadas fehacientemente (Quercus sp., P. pinaster, Abies sp. y Cistus sp.), se han realizado dataciones de 10 carbones correspondientes a los taxones que se han considerado de mayor interés para los propósitos de la investigación, en este caso Quercus sp., P. pinaster y Abies sp. Estas muestras cubren la totalidad de los niveles de muestreo diferenciados en la localidad objeto de estudio, siendo el nivel IV del que se ha datado un mayor número de carbones (4) debido al interés que ofrecían los taxones identificados. La cronología de las dataciones, tal y como se puede apreciar en el cuadro 2 , comprende un periodo de tiempo que abarca desde el inicio del Holoceno medio, hace algo más de 8.000 años (considerando la datación calibrada), hasta hace unos 250300 años (dataciones sub-actuales). En cualquier caso, cabe resaltar que las dataciones obtenidas se concentran en dos periodos de tiempo muy definidos: un lapso temporal

Cuadro 2. Resultados de las dataciones efectuadas mediante el análisis de ${ }^{14} \mathrm{C}$ y dataciones calibradas (AP) (95 \%).

Results of the dating made through the ${ }^{14} \mathrm{C}$ analysis and calibrated dating (BP) $(95 \%)$.

\begin{tabular}{ccccc}
\hline $\begin{array}{c}\text { Código } \\
\text { laboratorio }\end{array}$ & $\begin{array}{c}\text { Nivel de muestreo } \\
\text { profundidad en cm) }\end{array}$ & Taxón & $\begin{array}{c}\text { Datación }{ }^{14} \mathrm{C} \\
(\text { años AP) }\end{array}$ & $\begin{array}{c}\text { Datación calibrada } \\
(\text { años AP) }(95 \%)\end{array}$ \\
\hline Poz-78848 & I (0-9) & Quercus sp. & $140 \pm 30$ & $281-6$ \\
Poz-78849 & II (9-21) & Pinus pinaster & $70 \pm 30$ & $260-26$ \\
Poz-78850 & III (21-32) & Quercus sp. & $50 \pm 30$ & $257-31$ \\
Poz-78929 & III (21-32) & Quercus sp. & $90 \pm 30$ & $266-22$ \\
Poz-78851 & IV (32-52) & Abies sp. & $6.480 \pm 40$ & $7.470-7.310$ \\
Poz-78852 & IV (32-52) & Abies sp. & $5.710 \pm 40$ & $6.631-6.408$ \\
Poz-78853 & IV (32-52) & Quercus sp. & $120 \pm 30$ & $272-11$ \\
Poz-78854 & IV (32-52) & Pinus pinaster & $4.965 \pm 35$ & $5.854-5.604$ \\
Poz-78856 & V (52-80) & Quercus sp. & $135 \pm 30$ & $279-8$ \\
Poz-78857 & V (52-80) & Pinus pinaster & $7.300 \pm 40$ & $8.180-8.020$ \\
\hline
\end{tabular}


de unos 2.500 años que comprende cuatro muestras datadas hace entre 8.180 y 5.604 AP y, en segundo lugar, un periodo sub-actual de cronología inferior a 300 años que aglutina el resto de muestras datadas (6). Nótese el vacío temporal de registros encontrados entre 5.600 y $300 \mathrm{AP}$.

Las cuatro dataciones que integran el periodo cronológico más antiguo corresponden a Abies sp. (2) y a $P$. pinaster (2). Tres de estas muestras provienen de carbones encontrados en el nivel IV, correspondientes a Abies sp. (7.470-7.310 y 6.631-6.408 AP) y P. pinaster (5.854-5.604 AP); la cuarta muestra corresponde a un carbón encontrado en el nivel $\mathrm{V}$, en este caso de P. pinaster, siendo esta la de mayor antigüedad (8.180-8.020 AP). Destaca, asimismo, el hecho de que, junto las anteriores dataciones antiguas, se hayan datado dos muestras de carbón de edad sub-actual (270-280 años) (una en cada nivel de muestreo IV y V); esta circunstancia indica, a priori, la inexistencia de orden cronológico alguno en los distintos horizontes identificados en el corte edáfico analizado $\mathrm{y}$, por tanto, el verdadero funcionamiento de este suelo como un tipo de montaña sin características aluviales sedimentarias estrictas a pesar de situarse en el fondo de una pequeña cuenca endorreica. El resto de dataciones sub-actuales (4), que integran el periodo cronológico más moderno, corresponden a Quercus sp. para tres de las muestras (nivel I y III) y a $P$. pinaster para la cuarta (nivel II), las cuales ejemplifican a la perfección la vegetación actualmente existente en la cuenca. La figura 4 representa gráficamente la distribución temporal de estas dataciones considerando los tres taxones principales identificados (P. pinaster, Abies sp. y Quercus sp.).

\section{DISCUSIÓN}

La presencia en el registro fósil de carbón de Abies sp. en la localidad estudiada -con gran certeza perteneciente a $A$. pinsapo- confirma la existencia en el pasado de un área de distribución de la especie más extensa que la actual en el marco geográfico del extremo occidental de la Cordillera Bética en general y, en particular, en Sierra Bermeja. Y es que, a pesar de que la mayor parte de los carbones de coníferas analizados en la localidad objeto de estudio pertenecen a $P$. pinaster, se han podido identificar algunas muestras pertenecientes a Abies sp. gracias a una característica particular, en forma de "cremallera", diferenciable en el plano radial de los abetos pero inexistente en el mismo plano anatómico en los pinos (figura 5). La antedicha hipótesis ya había sido apuntada por Linares (2011) basándose en fósiles de ancestros comunes de distintas especies de abetos circunmediterráneos integrantes de bosques terciarios, cuya distribución biogeográfica fue mucho más amplia en el Mediterráneo Occidental. En el caso del sur de la Península Ibérica y norte de África, el área de distribución de los abetales se vio favorecida por las oscilaciones climáticas relacionadas con los continuos avances de los fríos glaciales, que propiciaron la contracción y fragmentación de los rangos de distribución de aquellas especies mejor adaptadas a climas templados (Van der Veken et al. 2007, Alba-Sánchez y López 2013). Por otro lado, las señales polínicas de abeto encontradas en determinadas lagunas, turberas y cuevas del sur y sureste de la Península Ibérica (véase Carrión 2013) respaldan

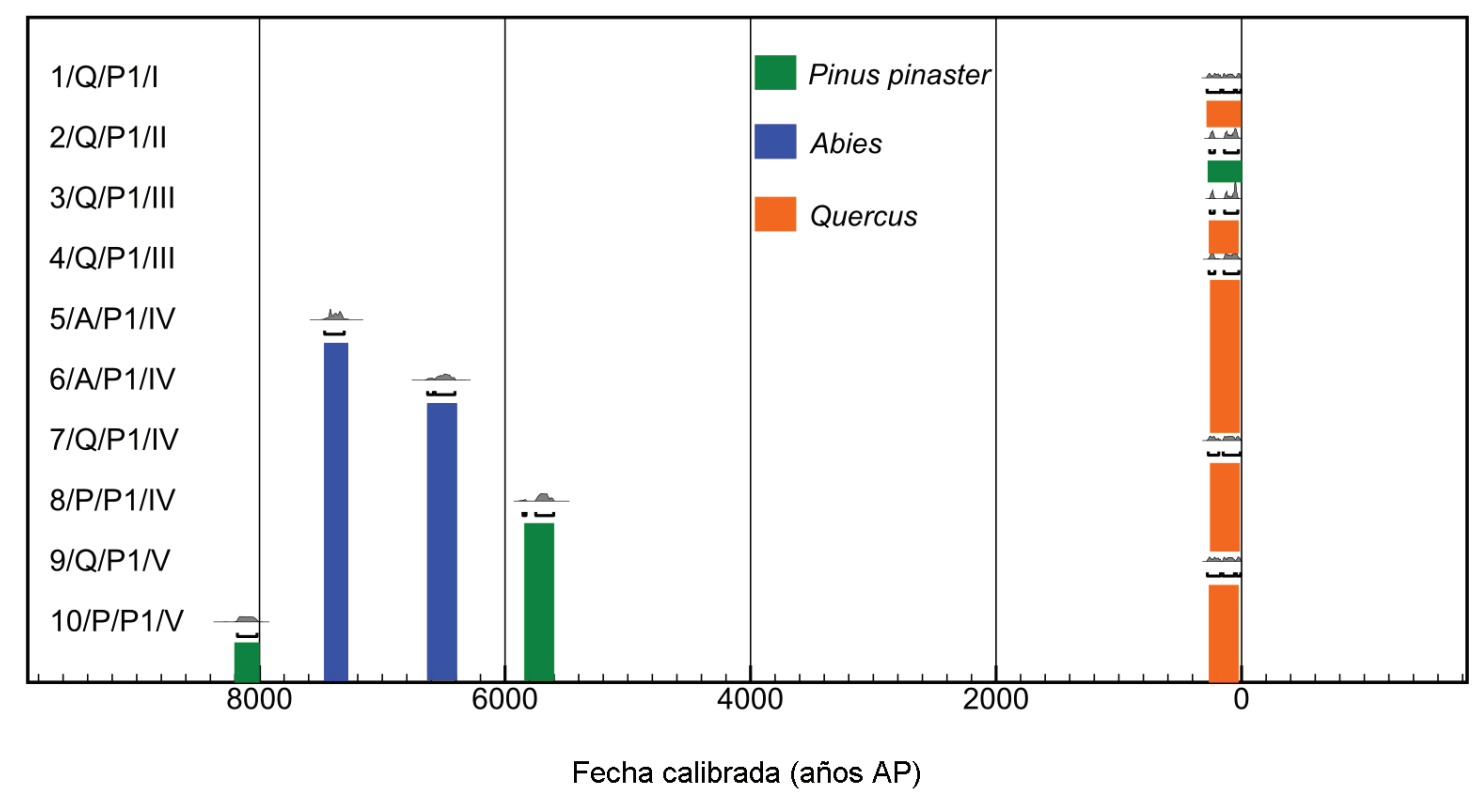

Figura 4. Distribución cronológica de las dataciones realizadas. Los diferentes colores representan los taxones correspondientes. Elaborado con el programa Oxcal 4.2 y la base de datos IntCal13 (Reimer et al. 2013), 2 sigma (95 \% probabilidad).

Chronological distribution of the obtained dating. The different colors represent the corresponding taxa. 

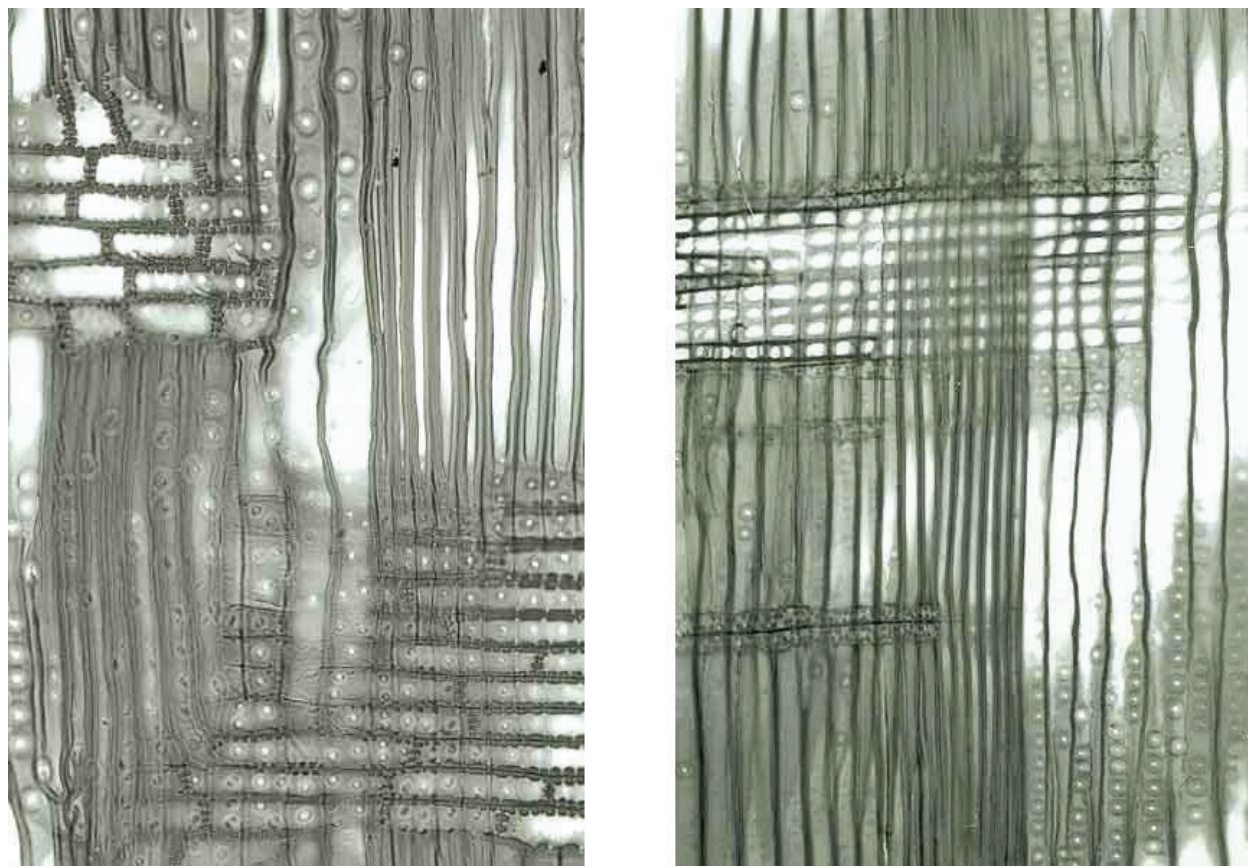

Figura 5. Imágenes de carbón al microscopio. Secciones radiales de Abies sp. (izquierda) y Pinus sp. (derecha). Obsérvese la "cremallera" del plano radial de Abies sp., y cómo dicha característica no aparece en la misma sección en Pinus sp. (Schoch et al. 2004).

Images of charcoal samples under a microscope. Radial sections of Abies (left) and Pinus (right). Note the "zipper" of the radial plane of Abies, and how this feature does not appear in the same section in Pinus. Source of the images: http://www.woodanatomy.ch/species_az.php.

esta hipótesis, llegándose a detectar registros de polen de Abies sp. superiores al 3-5\%, valores que se han establecido como el parámetro que asegura la presencia de los bosques in situ.

En este contexto, y considerando que en la actualidad solo se conservan abetales en el paraje de Los Reales dentro del conjunto petrográfico de Sierra Bermeja (figura 6, arriba), resulta de gran interés el presente hallazgo, en el registro fósil, de evidencias físicas de Abies sp., que asegura su presencia pretérita en una localidad en la que actualmente la especie se encuentra extinta, suponiendo un paso importante para el conocimiento de su paleobiogeografía en el sur de España. Asimismo, estos análisis pedoantracológicos, contrastados con el estudio de la vegetación actual, despejan prácticamente todas las dudas sobre la enquistada controversia existente hasta ahora entre latifolias y coníferas sobre los sustratos ultramáficos mediterráneos de Sierra Bermeja (Gómez-Zotano 2004) ya que confirman la antigüedad de las coníferas como vegetación climácica sobre los litosoles serpentínicos béticos, que se puede considerar se extiende al menos a lo largo de gran parte del Holoceno.

Asimismo, las dataciones de Abies sp. y P. pinaster obtenidas en la localidad de estudio permiten teorizar acerca de cómo pudo ser la dinámica vegetal local durante 8.100-5.600 AP. En este sentido, se detectan dos fases de preponderancia de los pinares respecto a los abetales, acotadas en torno a 8.100 y $5.700 \mathrm{AP}$, mientras que Abies sp. pudo ser dominante en la fase comprendida entre $7.400 \mathrm{y}$
6.400 AP. Esta dinámica vegetal teórica se enmarcaría en un fase dilatada de transformación del paisaje vegetal dominante en esta zona del sur de la Península Ibérica, que estaría muy emparentada con los cambios ambientales bien conocidos- acontecidos en la cercana y limítrofe región del Estrecho de Gibraltar; en este sector coexistían bosques húmedos integrados por los ancestros de taxones actualmente existentes como quejigos comunes (Quercus faginea Lam.) y andaluces (Quercus canariensis Willd.), robles melojos (Quercus pyrenaica Willd.), alcornoques (Quercus suber L.), quejiguetas (Quercus lusitanica Lam.), arces granadinos [Acer opalus Mill. ssp. granatense (Boiss.)], acebos (Ilex aquifolium L.), abedules [Betula pendula Roth ssp. fontqueri (Rothm.) Moreno y Peinado] y castaños (Castanea sativa Mill.) durante el Paleolítico final (bajo condiciones favorables de humedad y temperie creciente), que pudieron convivir o dejar paso a masas mixtas de Quercus sp. y Pinus sp. con Juniperus sp. en las fases progresivamente más secas del Holoceno inicial, cuyo paisaje vegetal experimentó una paulatina homogeneización florística (Carrión et al. 2008). Este proceso de simplificación de la flora forestal, vigente en la actualidad, se asocia en la Península Ibérica y norte de Marruecos al impacto antropogénico de incendios y ganadería durante el Neolítico (Reille 1977, Arroyo et al. 2004). Una temprana humanización que, en el entorno de Sierra Bermeja, se constata en el cercano paraje del Torcal de la Utrera (distante $28 \mathrm{~km}$ en línea recta de PALMI), donde se ha 


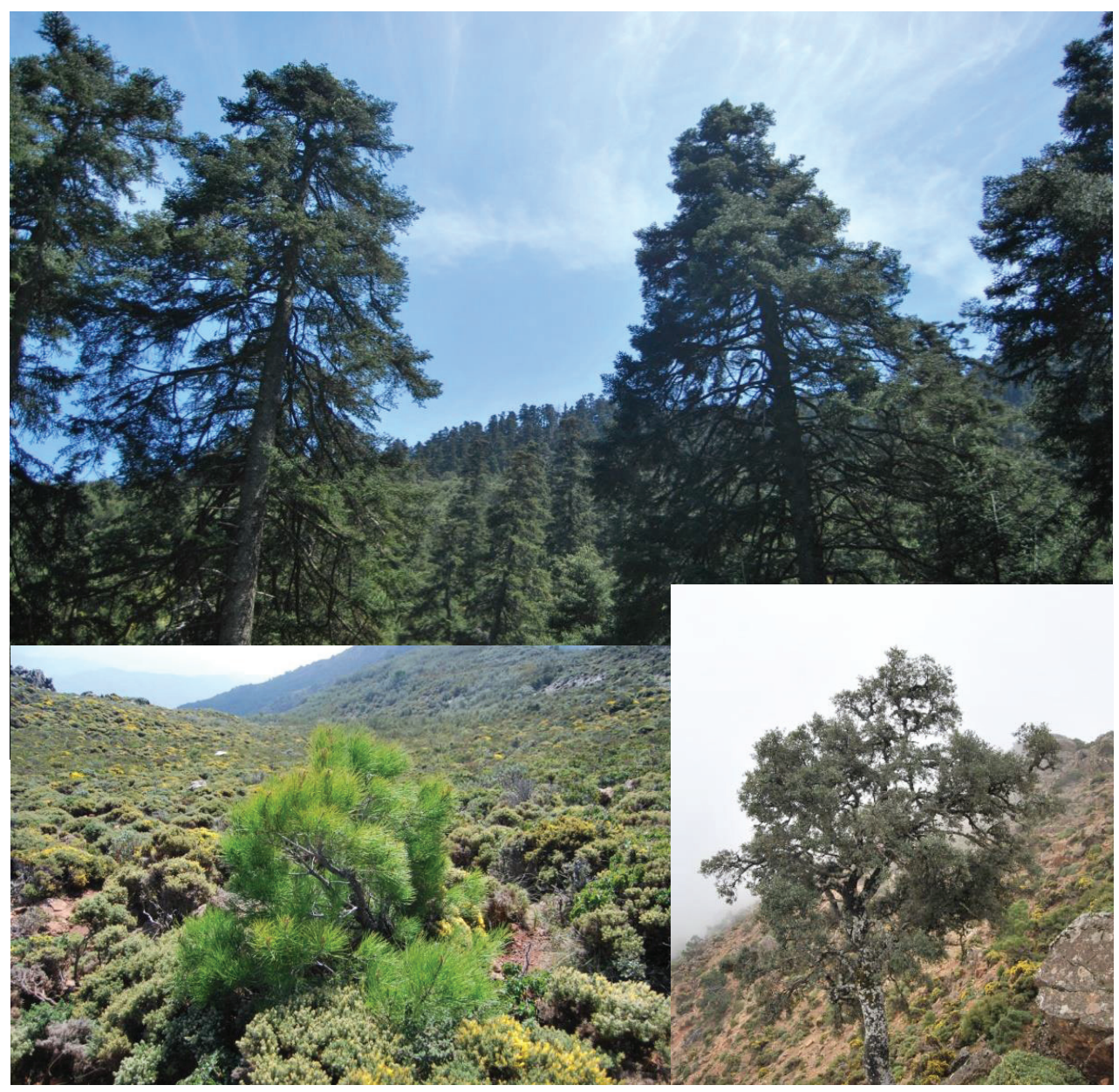

Figura 6. Abetal de A. pinsapo en Los Reales de Sierra Bermeja (arriba); P. pinaster joven en la localidad objeto de estudio (abajo izquierda); y $Q$. rotundifolia relictual en la crestería de la Sierra de la Palmitera sobre afloramiento de dique granítico.

Firs forest of Abies pinsapo in Sierra Bermeja (top); Young Pinus pinaster growing in the study area (bottom left); And relict Quercus rotundifolia in the upper crest of Sierra de la Palmitera over granite dikes outcrop (bottom right).

comprobado la existencia de un poblamiento neolítico especialmente intenso (8.000-6.000 AP) asociado a cavidades de origen kárstico (Romo et al. 2008).

Este proceso de neolitización en el litoral y prelitoral cercano a Sierra Bermeja parece que coincidió con el rápido desarrollo del clima mediterráneo al comienzo del Holoceno, según apuntan los análisis polínicos y los niveles lacustres (Cheddadi et al. 1998, Jalut et al. 2000), destacando las notables pulsaciones de aridez acaecidas desde entonces, si bien destaca entre ellas una acusada fase húmeda en el lapso 7.000-6.000 AP (Reed et al. 2001), coincidente en este caso con la presencia de Abies sp. en la zona de estudio. Por tanto, es previsible que durante el periodo 8.000-6.000 AP Sierra Bermeja en su conjunto estuviese sometida a unas condiciones ambientales progresivamente más secas y templadas. Se puede argüir que el carbón encontrado con dicha antigüedad se enmarcaría en un momento de intensificación de los incendios forestales al principio de dicho periodo, tanto de tipo natural (debido a la desecación climática) como de origen antropogénico (asociado al poblamiento neolítico en el cercano litoral), lo que habría supuesto una drástica, progresiva e imparable transformación vegetal de la montaña peridotítica. En cualquier caso, no es posible aseverar que $P$. pinaster sustituyese por completo a Abies sp., como tampoco es previsible que en 7.000-6.000 AP, bajo condiciones más húmedas, Abies sp. desplazase por entero a P. pinaster.

En este sentido, el hipotético aumento de la recurrencia e intensidad de los incendios forestales en Sierra Bermeja desde hace aproximadamente 8.000 años, junto a las características geomorfológicas de la localidad de estudio -fondo de una pequeña cuenca endorreica-, pueden ayudar a explicar las altas tasas de antracomasa encontradas, superiores a $4.000 \mathrm{mg} \mathrm{kg}^{-1}$ en todos los niveles de muestreo y por encima de $112.000 \mathrm{mg} \mathrm{kg}^{-1}$ en el nivel II. En los ambientes de montaña o media montaña pirenaicos, alpinos o 
centroeuropeos, las antracomasas específicas por nivel de muestreo raramente superan los $1.000 \mathrm{mg} \mathrm{kg}^{-1}$ (Touflan y Talon 2008, Bal et al. 2010, Cunill et al. 2015), y solo en el norte de Alemania se han encontrado niveles de alrededor de $9.000 \mathrm{mg} \mathrm{kg}^{-1}$ por nivel (Robin et al 2013).

En el caso del nivel II (antracomasa $>112.000 \mathrm{mg} \mathrm{kg}^{-1}$ ), es previsible que la acumulación de carbón en torno a 12$15 \mathrm{~cm}$ de profundidad provenga de incendios forestales relativamente recientes a la vista de las dataciones obtenidas de los carbones más superficiales analizados, que en todos los casos han ofrecido una cronología sub-actual inferior a 300 años; la eliminación en repetidas ocasiones de la cubierta arbolada y del matorral a causa del fuego habría dejado desnudas de vegetación las laderas y, en consecuencia, expuestos los delgados y rocosos suelos a la erosión hídrica de la capa fértil. Este proceso degenerativo del suelo habría sido particularmente importante durante los años inmediatamente posteriores a los episodios de fuego -cuando aún la regeneración vegetal era escasa- en relación a las intensas lluvias otoñales que caracterizan el mesoclima de Sierra Palmitera, con acumulados que frecuentemente superan los 50-100 mm día ${ }^{-1}$.Todavía en la actualidad, tras más de 40 años del último incendio que asoló la localidad, la cubierta vegetal es abierta en las posiciones de mayor pendiente, siendo algo más espesa hacia el fondo de la cuenca y, en cualquier caso, carente de cobertura arbolada alguna a excepción de algunos pies de $P$. pinaster supervivientes del último fuego.

A todo ello hay que añadir el hecho de que las especies a las que pertenecen estas muestras sub-actuales, P. pinaster y Quercus sp., corresponden a los taxones que actualmente protagonizan la cubierta vegetal de la localidad de estudio (figura 6, abajo izquierda y derecha, respectivamente), lo que parece indicar una drástica homogeneización de la vegetación coincidente con esta última y más atroz fase de incendios forestales que ha castigado Sierra Bermeja en su conjunto desde el siglo XVIII. Bien es cierto que la ausencia de dataciones que se ha obtenido entre 300 y $5.600 \mathrm{AP}$ abre una serie de incógnitas a resolver sobre la evolución del paisaje vegetal en Sierra Palmitera durante buena parte del Holoceno reciente, si bien se puede plantear como causa probable de este hecho un notable descenso en la frecuencia de los incendios forestales, lo que en cualquier caso debería ser corroborado mediante otras técnicas de análisis paleoecológicas.

Además, en el caso de Quercus sp., la imposibilidad de poder discernir a qué especie corresponden las muestras identificadas plantea un escenario interesante a la hora de analizar si la coscoja (Quercus coccife$r a$ L.) -el más extendido de los axones que integran el género Quercus en las peridotitas suribéricas- ha estado presente en Sierra Bermeja a lo largo del Holoceno de manera coetánea a $P$. pinaster o, si por el contrario, Q. rotundifolia tuvo un papel más principal en el pasado; asimismo, la posible coexistencia entre ambas especies debe considerarse como una posibilidad, al menos en aquellos ni- chos ecológicos donde $Q$. rotundifolia aparece en la actualidad con mayor profusión, que en todo caso corresponden a situaciones lito-edáficas y topográficas particulares como afloramientos de rocas ácidas (diques graníticos) y vaguadas profundas, hondonadas y umbrías donde los suelos de origen peridotítico han logrado alcanzar un grado considerable de profundidad.

Como cuestiones complementarias a los resultados principales obtenidos, merece la pena resaltar para finalizar dos circunstancias. En primer lugar, la elevada tasa de carbón vitrificado que no ha podido ser identificado (casi un tercio del total de las muestras analizadas) debe ser tenida en cuenta al realizar distintos tipos de modelizaciones basadas en las especies identificadas en la localidad de estudio. La vitrificación de los carbones, cuyas causas no han podido todavía ser determinadas de manera general (y así queda recogido en la literatura científica), podría en este caso maximizar o minimizar la importancia de los principales taxones arbóreos identificados-Abies, Pinus o Quercus - o de otras nuevas especies, que podrían hacer variar en distinto grado las conclusiones que emanan de este trabajo. En segundo lugar, los resultados obtenidos demuestran la inexistencia de una estratificación cronológica completa en el suelo analizado, ya que las muestras de carbón de los niveles IV y V presentan edades comprendidas entre la actualidad (sub-actuales) y 8.180 AP. Frente a esta "mezcla" cronológica, la parte superior del suelo (niveles I, II y III) sí parece resultado de procesos edafogenéticos recientes, pues todas las muestras analizadas datan de hace relativamente poco tiempo. Considerando que es bien conocida la inexistencia de relación directa entre la edad de los carbones y su profundidad en los suelos de montaña, dada la intervención de diferentes procesos edafológicos y de bioturbación (Carcaillet y Talon 1996), se puede deducir que el suelo de la localidad objeto de estudio se ha formado sobre un lecho de origen coluvial que facilita la infiltración del agua y que, pese a la inexistencia de sumidero, no presenta indicios de encharcamiento (cuarteamiento $\mathrm{u}$ horizonte gris del suelo, vegetación higrófila).

\section{CONCLUSIONES}

Los resultados obtenidos del análisis pedoantracológico en la localidad objeto de estudio confirman, una vez contextualizados con aquellos derivados del resto de estudios paleoambientales, la existencia en el pasado de un área de distribución de Abies sp. en la Cordillera Bética (sur de la Península Ibérica) más extensa que la actual y sobre peridotitas. El hallazgo de carbón de Abies sp. - perteneciente muy probablemente a $A$. pinsapo- en el registro fósil de Sierra Palmitera, una localidad de Sierra Bermeja donde actualmente los abetales están ausentes, aporta, sin duda, un conocimiento fundamental para el estudio paleobotánico de este paleoendemismo ibérico. Las dataciones antiguas de esta conífera, así como las correspondientes a $P$. pinaster, vienen a solventar en gran medida la hasta 
ahora enquistada controversia biogeográfica latifolias-coníferas sobre los sustratos ultramáficos de Sierra Bermeja, quedando meridianamente claro que pinares y abetales constituyen la vegetación climácica de la práctica totalidad del macizo, lo que se puede hacer extensible a gran parte del Holoceno.

El mayor conocimiento paleocológico de la biogeografía holocena de Abies sp. y del papel paleobiogeográfico que han tenido los bosques de gimnospermas, de los que forman parte los abetales, frente a las formaciones de angiospermas de Quercus sp. debe resultar esencial para la adecuada gestión actual de $A$. pinsapo. La información colectada ha de implementarse necesariamente con las medidas que actualmente se están llevando a cabo (lucha y prevención de incendios forestales, vigilancia y control fitosanitario de agentes patógenos, reforestación y reforzamiento de abetales residuales y de poblaciones con escasa capacidad de regeneración natural, actuaciones de mantenimiento y/o de mejora de hábitats, preservación del acervo genético del pinsapo y control de sus poblaciones frente a las amenazas del cambio global, además de acciones de educación y concienciación social acerca de la importancia que tiene esta especie) para conformar una nueva estrategia realista con el objeto de la preservación y regeneración de la especie -dado su estatus en peligro de extinción- basada en el conocimiento de su evolución desde el Holoceno medio hasta el presente. En este sentido, los datos obtenidos avalan la posibilidad de reintroducir el pinsapar en aquellos enclaves considerados nichos ecológicos potenciales de $A$. pinsapo, y no solo en los tres núcleos disyuntos de montaña donde se refugia, sierras de las Nieves, Grazalema y Bermeja, sino también allí donde los análisis pedoantracológicos y demás afines paleoecológicos determinen que existió idoneidad para ello. En cualquier caso, la gran cantidad de carbón vitrificado que no ha podido ser identificado plantea todavía cuestiones a resolver sobre la posibilidad de que determinadas especies presenten mayor o menor importancia que la que parecen tener a partir de las dataciones efectuadas.

\section{AGRADECIMIENTOS}

Los resultados de esta investigación forman parte del Proyecto de I+D "Reconstrucción geohistórica de la vegetación arbórea sobre sustratos ultramáficos mediterráneos" (ULTRAFOERSTS - CSO2013-47713-P), perteneciente al Programa Estatal de Investigación Científica y Técnica de Excelencia - Subprograma Estatal de Generación de Conocimiento, subvencionado por el Ministerio de Economía y Competitividad del Gobierno de España (Convocatoria 2013).

\section{REFERENCIAS}

Alba-Sánchez F, JA López. 2013. Paleobiogeografía del pinsapo en la Península Ibérica durante el Cuaternario. In López C ed. Los Pinsapares en Andalucía (Abies pinsapo Boiss.): Conservación y sostenibilidad en el siglo XXI. Sevilla, España. Junta de Andalucía, Consejería de Agricultura, Pesca y Medio Ambiente. p. 21-39.

Alcalde C, I García-Amorena, S García Álvarez, D García Calvo, R García García, M Génova, P Gil Borrell, F Gómez Manzaneque, J Maldonado, C Morla, J del Nido, JM Postigo, P Regato, S Río, S Roig, JM Rubiales, LJ Sánchez. 2006. Contribución de la Paleofitogeografía a la interpretación del paisaje vegetal ibérico: estado de conocimientos y nuevas perspectivas de investigación. Investigación Agraria. Sistemas y Recursos Forestales Fuera de serie: 40-54.

Arroyo J, J Carrión, A Hampe, P Jordano. 2004. La distribución de las especies a diferentes escalas espacio-temporales. In Valladares F ed. Ecología del bosque mediterráneo en un mundo cambiante. Madrid, España. Editorial EGRAF S.A. p. 29-69.

Asensi A, S Rivas-Martínez. 1976. Contribución al conocimiento fitosociológico de los Pinsapares de la Serranía de Ronda. Anales del Instituto Botánico Antonio José Cavanilles 33:239-247.

Asensi A, B Diez-Garretas. 1992. Ecología y siataxanamía de los matorrales y jarales de la Serranía de Rauda (Andalucía, España). Doc. Pbyíasuciol 13:15-28.

Bal MC, C Rendu, MP Ruas, P Campmajo. 2010. Paleosol charcoal: reconstructing vegetation history in relation to agropastoral activities since the Neolithic. A case study in the Eastern French Pyrenees. Journal of Archaeological Science 37(8): 1785-1797.

Carcaillet C, B Talon. 1996. Aspects taphonomiques de la stratigraphie et de la datation de charbons de bois dans les sols: exemple de quelques sols des Alps. Géographie Physique et Quaternaire 50(2): 233-244.

Carcaillet C, M Thinon. 1996. Pedoanthracological contribution to the study of the evolution of the upper treeline in the Maurienne Valley (North French Alps): methodology and preliminary results. Review of Palaeobotany and Palynology 9: 399-416.

Carrión J ed. 2013. Paleoflora Ibérica: Plioceno-Cuaternario (Vol. I-II). Madrid, España. Ministerio de Economía y Competitividad. $543 \mathrm{p}$.

Carrión J, C Finlayson, S Fernández, G Finlayson, E Allué, J López-Sáez, P López-García, G Gil-Romera, G Bailey, P González-Sampériz. 2008. A coastal reservoir of biodiversity for Upper Pleistocene human populations: palaeoecological investigations in Gorham's Cave (Gibraltar) in the context of the Iberian Peninsula. Quaternary Science Reviews 27: 2118-2135.

Cheddadi R, HF Lamb, J Guiot, S van der Kaars. 1998. Holocene climatic change in Morocco: a quantitative reconstruction from pollen data. Climate Dynamics 14: 883-890.

Cunill R, JP Métailié, D Galop, S Poublanc, N de Munnik. 2015. Palaeoecological study of Pyrenean lowland fir forests: Exploring mid-late Holocene history of Abies alba in Montbrun (Ariège, France). Quaternary International 366: 37-50.

Gómez-Zotano J. 2004. Controversia árboles latifolios-versus coníferas en Sierra Bermeja, una montaña ultramáfica del sur de España. In Boyd RS, AJM Baker, J Proctor eds. Ultramafic Rocks: Their Soils, Vegetation, and Fauna. St. Albans-Herts, United Kingdom. p. 151-156.

Gómez-Zotano J, F Román-Requena, N Hidalgo-Triana, AV Pé- 
rez-Latorre. 2014. Biodiversidad y valores de conservación de los ecosistemas serpentínicos en España: Sierra Bermeja (provincia de Málaga). Boletín de la Asociación de Geógrafos Españoles 65: 187-206.

Gómez-Zotano J, JA Olmedo-Cobo, E Martínez-Ibarra, R CunillArtigas. 2015. Descubrimiento y caracterización de una cuenca endorreica en la cumbre de Sierra Bermeja (provincia de Málaga). In De la Riva J, P Ibarra, R Montorio, M Rodrigues eds. Análisis espacial y representación geográfica: innovación y aplicación. Zaragoza, España. Editorial ISBN. p. 1629-1638.

Jalut G, AE Amat, L Bonnet, T Gauquelin, M Fontugne. 2000. Holocene climatic changes in the Western Mediterranean, from south-east France to south-east Spain. Palaeogeography, Palaeoclimatology, Palaeoecology 160: 255-290.

Linares JC. 2011. Biogeography and evolution of Abies (Pinaceae) in the Mediterranean Basin: the roles of long-term climatic change and glacial refugia. Journal of Biogeography 38: 619-630.

Mota JF, JM Medina-Cazorla, FB Navarro, FJ Pérez-García, AV Pérez-Latorre, P Sánchez-Gómez, JA Torres, A Benavente, G Blanca, C Gil, J Lorite, ME Merlo. 2008. Dolomite flora of the Baetic Ranges glades (South Spain). Flora 203: 359-375.

Reed JM, AC Stevenson, S Juggins. 2001. A multi-proxy record of Holocene climatic change in SW Spain: Laguna de Medina, Cádiz. The Holocene 11(6): 707-719.

Reille M. 1977. Contribution pollen anàlytique à l'histoire holocène de la vegetation des montagnes du Rif (Maroc Septentrional). Recherches Françaises sur le Quaternaire 50: 53-76.

Reimer PJ, E Bard, A Bayliss, JW Beck, PG Blackwell, CB Ramsey, CE Buck, H Cheng, RL Edwards, M Friedrich, PM
Grootes, TP Guilderson, H Haflidason, I Hajdas, C Hatté, TJ Heaton, DL Hoffmann, AG Hogg, KA Hughen, KF Kaiser, B Kromer, SW Manning, M Niu, RW Reimer, DA Richards, EM Scott, JR Southon, RA Staff, CSM Turney, J van der Plicht. 2013. IntCal13 and Marine13 radiocarbon age calibration curves $0-50,000$ years cal BP. Radiocarbon 55(4): 1869-1887.

Rivas-Martínez S. 2011. Memoria del Mapa de Vegetación Potencial de España. Itinera Geobotanica 18(1-2): 5-800.

Robin V, B Talon, O Nelle. 2013. Pedoanthracological contribution to forest naturalness assessment. Quaternary International 289: 5-15.

Romo JL, J Gómez-Zotano, JI Torres-Díaz, G Torres-Díaz. 2008. Exploraciones subterráneas en el Karst de la Utrera (Casares, Málaga). In Diputación de Córdoba-Ediciones y Junta de Andalucía ed. Priego de Córdoba, España. p. 89-113.

Schoch W, I Heller, FH Schweingruber, F Kienast. 2004. Wood anatomy of Central European Species. WSL - Swiss Federal Institute for Forest, Snow and Landscape Research. Consultado 13 ene. 2017. Disponible en http://www.woodanatomy.ch/species

Talon B, C Carcaillet, M Thinon. 1998. Études pédoanthracologiques des variations de la limite supérieure des arbres au cours de 1'Holocene dans les Alps françaises. Géographie Physique et Quaternaire 52: 195-208.

Touflan P, B Talon. 2008. Étude pédoanthracologique à haute résolution spatiale de l'histoire holòcene d'une forêt subalpine (Alpes du Sud, France). Ecologia Mediterranea 34: 13-23.

Van der Veken S, J Bellemare, K Verheyen, M Hermy. 2007. Life-history traits are correlated with geographical distribution patterns of western European forest herb species. Journal of Biogeography 34: 1723-1735. 